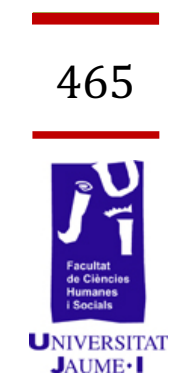

\title{
El rendimiento académico musical: el efecto de la creatividad, lateralidad e inteligencia musical.
}

Gustau Olcina Sempere Universitat Jaume I golcina@uji.es

César Carlos Ávila Rivera Universitat Jaume I avila@psb.uji.es 
El presente estudio tiene como principal objetivo conocer cómo influye la creatividad en el rendimiento académico en Música. De este objetivo principal se extraen los siguientes objetivos específicos: Analizar la relación existente entre la creatividad con la lateralidad cerebral por un lado, y con la inteligencia musical por el otro, y examinar la relación entre la creatividad, la lateralidad y los resultados académicos en música.

Para alcanzar estos objetivos se ha utilizado una metodología no experimental, ya que a los alumnos no se les ha aplicado ningún tratamiento antes de recabar los datos.

Los resultados revelan que la creatividad, la lateralidad y la inteligencia musical se asocian positivamente con el rendimiento musical, pero esta relación no es estadísticamente significativa.

Este trabajo concluye que la creatividad, la lateralidad y la inteligencia musical no son factores que expliquen el rendimiento académico musical, lo que implica que se tiene que seguir investigando para poder conocer qué factores determinan el rendimiento académico musical.

Palabras clave: Creatividad, dominancia lateral, inteligencia musical, rendimiento académico en Música.

\section{Introducción}

El conocimiento de la creatividad en el ámbito educativo, laboral y social ha sido y es una fuente de estudio e interés para la sociedad en la que vivimos, de forma que éste fomenta la necesidad de estudiar dicho aspecto con la finalidad de poder establecer unas líneas de actuación que contribuyan a su desarrollo e interés tanto desde el punto de vista individual como grupal.

El interés por conocer la influencia de la creatividad en la educación debe proporcionar unas herramientas útiles para el desarrollo de ésta en el aula, de manera que ayudemos a nuestros alumnos a aprender de manera creativa, y que su aprendizaje en los diferentes ámbitos educativos contribuya a desarrollar en sus diferentes contextos sociales un espíritu donde la motivación les conduzca a luchar contra la rutina y el aburrimiento, entre otros, y les anime a sumergirse en el mundo de lo desconocido. Con ello, se pretende contribuir a mejorar el desarrollo de la personalidad de los alumnos.

Así pues, el objetivo principal de este estudio es analizar la relación existente entre la creatividad de los alumnos de 3 o curso de Educación Primaria y el rendimiento académico que obtienen en música; más concretamente se quiere estudiar si la creatividad predice el rendimiento académico. De este objetivo principal se extraen los siguientes objetivos específicos: Analizar la relación existente entre la creatividad con la lateralidad cerebral por un lado, y con la inteligencia musical por el otro, y 
examinar la relación entre la creatividad, la lateralidad y los resultados académicos en música.

El interés que motiva el estudio de la relación entre la creatividad y el rendimiento académico en Música se justifica por la necesidad de conocer si debemos fomentar la creatividad de los alumnos para obtener mejores resultados en Música.

Teniendo en cuenta las consideraciones realizadas, es necesario conocer si existen dichas relaciones entre creatividad y resultados académicos, para después poder desarrollar programas que contribuyan a promover la creatividad tanto en el ámbito educativo como social, de manera que el fomento de la creatividad ayude a desarrollar una nueva forma de actuar en los distintos entornos sociales.

\section{Objetivos}

El presente estudio empírico plantea como principal objetivo analizar la relación existente entre la creatividad, la lateralidad, y la inteligencia musical de los alumnos de 30 de Educación Primaria con el rendimiento académico que obtienen en música. Concretamente, se quiere estudiar si la creatividad y la lateralidad predicen el rendimiento académico musical.

El objetivo general se divide en dos objetivos específicos. El primero de ellos relaciona, por una parte, la creatividad con la lateralidad, y por otra, la creatividad con la inteligencia musical, mientras que el segundo se centra en conocer la relación entre la creatividad y la lateralidad con los resultados académicos.

Respecto a la relación de la creatividad con la lateralidad y con la inteligencia musical, se trata de analizar la relación existente entre la creatividad con la lateralidad cerebral por un lado, y con la inteligencia musical, por otro.

Respecto a la relación de la creatividad y la lateralidad con los resultados académicos en música, se trata de examinar la relación existente entre la creatividad, la lateralidad y los resultados académicos en música.

En orden a alcanzar el primer objetivo de este estudio, se formula la siguiente hipótesis:

Hipótesis 1: Existe una relación directa y positiva entre la creatividad, la lateralidad manual izquierda, y la inteligencia musical.

Con la finalidad de obtener el segundo objetivo, se formulan las dos hipótesis siguientes:

Hipótesis 2: Los alumnos con mayor puntuación en creatividad y una lateralidad manual izquierda, obtendrán mejores resultados académicos

Hipótesis 3 : El tipo de lateralidad y la creatividad conjuntamente van a predecir el nivel de los resultados académicos. 
Como hemos descrito en los apartados anteriores, dicha investigación nos conduce a llevar a cabo una metodología no experimental, o conocida también con el nombre de "ex-post facto", es decir, aquella en la que el investigador recoge los datos después de haber sucedido los hechos y trata de extraer las posibles relaciones entre ellos e incluso la capacidad predictiva (que no casualidad) de una variables sobre otras.

Para realizar este estudio de investigación, se utiliza la metodología cuantitativa siendo ésta de tipo descriptivo-relacional, ya que los datos recogidos nos describen los aspectos relacionados con la creatividad, la lateralidad y el rendimiento académico y se trata de poner en relación dichos datos.

Esta investigación se caracteriza por la ausencia de control del investigador sobre las variables independientes, debido a que dichas variables (creatividad, lateralidad y rendimiento académico en Música), no han sido manipuladas por el investigador antes de recoger los resultados, así como también por la rigurosidad y sistematicidad en el proceso de investigación.

Para llevar a cabo la recogida de los datos, ésta se realiza en un único momento dentro del propio centro educativo, a fin de evitar variables extrañas que pudieran afectar a las respuestas dadas por los sujetos (efecto de aprendizaje sobre el tema, eventos personales que pudieran afectar a la concentración o respuestas de los sujetos, etc.).

La aplicación de las diferentes pruebas se realizará a primera hora de la mañana, con la intención de evitar la fatiga acumulada durante el día. También se ha realizado este control de las variables extrañas que puedan afectar al estudio o control de amenazas a la validez de resultados indicados, con la intención de controlar la varianza sistemática o secundaria.

En esta investigación hablamos de un diseño de "ciego único", el cual tiene la finalidad de no revelar a los alumnos el propósito de la investigación, para que los alumnos no tiendan a confirmar o rechazar las hipótesis planteadas en la investigación (control del efecto de deseabilidad social).

Además, se ha minimizado la varianza de error, eligiendo a grupos homogéneos de alumnos, ya que todos los alumnos pertenecían al mismo nivel académico, eran de la misma edad, y tenían el mismo nivel de rendimiento académico en cada uno de los grupos.

Así pues, por todo lo dicho anteriormente, nuestro diseño se define como un diseño ex post facto, correlacional y de corte transversal. 


\section{1.- Población y muestra}

La muestra que vamos a utilizar en este estudio está formada por niños y niñas de 3 o de educación primaria. Concretamente, por dos grupos de 30 alumnos cada uno, los cuales han sido clasificados en función del rendimiento en la asignatura de Música, obteniendo un grupo de alto rendimiento y otro de bajo rendimiento. Para ello, se han tenido en cuenta las calificaciones de música del tercer trimestre del presente curso escolar.

El colegio donde se ha llevado a cabo el estudio está situado en el extrarradio de una gran ciudad con un entorno socio-económico y cultural bajo, debido a los diferentes fenómenos migratorios que se han sucedido en la zona objeto de estudio. El principal motor económico de la zona en la que está ubicado el colegio son las pequeñas empresas de autónomos y los trabajos poco cualificados.

En la tabla 1 que se presenta a continuación ofrecemos los estadísticos descriptivos de la muestra objeto de este estudio.

Tabla 1. Anál isis descrip tivo de la muestra

Panel A. Variables continúas

\begin{tabular}{|c|c|c|c|c|c|c|}
\hline \multicolumn{7}{|l|}{ Variables } \\
\hline & $\mathbf{N}$ & Media & Mediana & Típica & Percentil 25 & Percentil 75 \\
\hline EDAD & 60 & 8.570 & 9.000 & 0.500 & 8.000 & 9.000 \\
\hline REND_ACD_MUS & 60 & 6.550 & 6.000 & 1.443 & 6.000 & 7.000 \\
\hline CREATIVIDAD & 60 & 11.370 & 10.000 & 4.769 & 8.000 & 14.750 \\
\hline INT_MUSI & 60 & 4.030 & 4.000 & 1.057 & 3.000 & 5.000 \\
\hline
\end{tabular}

Panel B. Variables dicotómicas

\begin{tabular}{lcccc}
\hline Variables & 0 & $\%(0)$ & 1 & $\%(1)$ \\
\hline SEXO & 33 & $55 \%$ & 27 & $45 \%$ \\
LATER & 4 & $7 \%$ & 56 & $93 \%$ \\
\hline
\end{tabular}

EDAD recoge los años que tienen los alumnos y alumnas que participan en la muestra del estudio.

REND_ACD_MUS es el rendimiento académico en música que han obtenido los alumnos en el tercer trimestre de este curso lectivo, medido mediante la nota obtenida en el tercer trimestre que puede oscilar entre 0 y 10.

CREATIVIDAD es la variable que muestra la creatividad, medida mediante el test de inteligencia creativa (CREA). El valor de esta variable resulta de la suma de un total de

20 preguntas que podían plantear los/as alumnos/as en función de la visualización de una imagen.

INT_MUSI es la variable que refleja la inteligencia musical, medida mediante el test de inteligencia musical de las inteligencias múltiples de 
Gardner (1983). Concretamente, se plantearon un total de 5 preguntas de índole musical y el total de síes acumulados daba lugar al resultado final de esta variable

SEXO es una variable dicotómica que tomará el valor 1 si el individuo de la muestra es un niño y 0 , si es una niña.

LATER es la variable que expresa la lateralidad, medida mediante el test de dominancia lateral de Harris (1993). Concretamente, si la dominancia lateral es diestra la variable toma el valor 1 y si es zurda toma el valor 0 .

Tal y como se puede apreciar en la tabla 1 , el total de miembros que forman la muestra de este estudio es de 60 , de los cuales un $45 \%$ son niños y un $55 \%$ son niñas. Así pues, podemos concluir que la muestra está equilibrada en cuanto a la participación femenina y masculina. Por otra parte, también puede observarse en la tabla 1 que la edad media de los/as alumnos/as es 8.5 años y el rendimiento medio académico musical (REND_ACD_MUS) es 6.55, por lo que podemos concluir que los/as alumnos/as de la muestra obtienen un bien alto en rendimiento académico musical. Con respecto a la lateralidad, también podemos apreciar que el 93\% de los individuos de la muestra tienen una lateralidad diestra, mientras que el $7 \%$ restante tienen una lateralidad zurda. Ello implica que en la gran mayoría de los miembros de la muestra predomina la dominancia lateral diestra y por tanto, utilizan para sus actividades académicas y cotidianas el ojo, el oído, la mano y el pie derecho. Si nos centramos en la creatividad, en la tabla 1 se puede apreciar que los/as alumnos/as de la muestra obtienen una puntuación de 11.37 en creatividad, lo que implica que, en media, estos/as alumnos/as han planteado más de la mitad de las preguntas que se les permitía, de un total de 20. Por tanto, podemos destacar que la creatividad de los/as alumnos/as de la muestra es media, a tenor de los resultados obtenidos. Finalmente, en lo referente a la variable inteligencia musical (INT_MUSI), los datos arrojan un valor medio de 4.03, revelando que los/as alumnos/as de la muestra presentan una elevada inteligencia musical, ya que de 5 preguntas planteadas en el test, respondieron afirmativamente, en media, a 4.03 preguntas.

\section{2.- Instrumentos de medida.}

Para llevar a cabo esta investigación, se utilizan los siguientes instrumentos de medida para la obtención de los datos:

Para conocer la variable de creatividad se emplea el cuestionario de Inteligencia Creativa (CREA) de los autores Corbalán et al. (2003).

El cuestionario de Inteligencia Creativa (CREA), consiste en realizar preguntas breves sobre la ilustración que se les presenta a los alumnos, pudiendo realizar hasta un máximo de 20 preguntas. Los niños se agrupan en dos grupos de 30 alumnos y se realiza la prueba de manera escrita. Está destinada para alumnos de 9 años, disponiendo de quince minutos para realizarla. 
En relación a la fiabilidad y validez de dicho test, se ha utilizado dos tipos de formatos, uno para adolescentes y otro para adultos, de manera que una vez analizados los resultados, se obtiene una fiabilidad del test del 0'875. Dicho dato, nos indica que el instrumento de medida de la creatividad se ajusta a los estándares actuales, y pone de manifiesto que la prueba mide con estabilidad y confianza la variable de la creatividad.

Para conocer la variable de lateralidad, se utiliza el test de dominancia lateral de Harris (1993).

El test de dominancia lateral de Harris, consiste en una serie de test de dominancia lateral, donde las puntuaciones obtenidas indican una mayor o menor preponderancia del dominio lateral. La prueba se realiza de manera individual a cada alumno, con una duración de 5 minutos para cada alumno.

Dicho test nos ofrece unas tareas que nos ayudan a conseguir los objetivos planteados en esta investigación. Además, el test se ha comparado con otros instrumentos de medida de las mismas características, obteniendo unos resultados favorables en cuanto su validez, permitiendo también su puesta en práctica, el poder establecer diferencias entre grupos ya formados.

Para conocer la variable de Inteligencia musical, se utiliza el cuestionario de las Inteligencias Múltiples de Gardner (1983).

El test para medir la inteligencia musical consta de cinco preguntas relacionadas con la música, del test de las inteligencias múltiples de Gardner. Los niños se agrupan en dos grupos de 30 alumnos y se realiza la prueba de manera escrita. Está destinada para alumnos de 9 años, y el tiempo para realizarla es de diez minutos.

Las investigaciones realizadas sobre la fiabilidad y validez del test de las inteligencias múltiples de Gardner, nos demuestran que el análisis factorial reproduce de manera clara la estructura de las inteligencias múltiples.

Para conocer la variable de rendimiento académico en Música, se utiliza la nota media obtenida en la asignatura de música durante el tercer trimestre del curso académico en el que se realiza la prueba.

La nota obtenida es el resultado de la evaluación continua en dicha asignatura, y se escoge ésta por ser la calificación más cercana a la fecha en que se mide con el resto de instrumentos de medida, como por ser aquella en la cual se refleja el grado de consecución de los objetivos, contenidos y criterios de evaluación planteados en dicho curso académico.

\section{Resultados}

A continuación presentamos los resultados para poder contrastar empíricamente las hipótesis planteadas. Para la primera hipótesis planteada ofrecemos los coeficientes de correlación de Pearson entre variables, para conocer la correlación entre las variables estudiadas, así como un análisis univariante de diferencia de medias, con la finalidad de 
analizar si hay diferencias estadísticamente significativas entre la diferencia de medias que presentan las variables analizadas.

Para la segunda hipótesis del estudio, ofrecemos dos análisis univariante de diferencia de medias para conocer la significatividad que presentan las variables estudiadas. Para contrastar la tercera hipótesis planteada, con la que se pretende conocer la capacidad predictiva de la lateralidad y la creatividad conjuntamente sobre el rendimiento académico, presentamos los resultados de la regresión lineal, del $\mathrm{R}$ cuadrado y de la técnica ANOVA.

En la tabla 2 presentamos los coeficientes de correlación de Pearson para contrastar empíricamente si existe una relación directa y positiva entre creatividad e inteligencia musical, tal y como se ha planteado en la hipótesis primera.

Tabla 2. Coeficientes de correlación de Pearson entre las variables de creatividad e inteligencia musical

\begin{tabular}{|c|c|c|}
\hline & CREATIVIDAD & INT_MUSI \\
& $\mathbf{N}$ & $\mathbf{N}$ \\
& $(\boldsymbol{p})$ & 0.253 \\
\hline CREATIVIDAD & 1 & 60 \\
& 60 & $(0.051)$ \\
\hline INT_MUSI & 0.253 & 1 \\
& 60 & 60 \\
\hline
\end{tabular}

CREATIVIDAD es la variable que muestra la creatividad, medida mediante el test de inteligencia creativa (CREA). El valor de esta variable resulta de la suma de un total de 20 preguntas que podían plantear los/as alumnos/as en función de la visualización de una imagen.

INT_MUSI es la variable que refleja la inteligencia musical, medida mediante el test de inteligencia musical de las inteligencias múltiples de Gardner (1983). Concretamente, se plantearon un total de 5 preguntas de índole musical y el total de síes acumulados daba lugar al resultado final de esta variable.

$\mathbf{N}$ nos indica el número de alumnos que forman la muestra.

$(p)=p$-value

Así como puede apreciarse en la tabla 2, existe una correlación positiva (0.253) y directa entre la variable creatividad e inteligencia musical, y por tanto, este resultado sugiere que a mayor inteligencia musical, mayor es también la creatividad del alumno. Ahora bien, esta correlación no es estadísticamente significativa (0.051), ya que el $p$-value es ligeramente $>.05$. 
En la tabla 3 presentamos las diferencias de medias que se ponen de manifiesto para las variables creatividad e inteligencia musical en base a la lateralidad manual izquierda. Para ello se han creado dos grupos: uno formado por aquellos/as alumnas/os que presentan una lateralidad diestra y otro formado por aquellos/as alumnos/as que presentan una lateralidad zurda, con el propósito de conocer si los alumnos más creativos y con más inteligencia musical presentan lateralidad zurda o diestra.

Tabla 3. Diferencia de medias para las variables creatividad e inteligencia musical en base a la lateralidad

\begin{tabular}{|c|c|c|c|c|}
\hline Variable & $\begin{array}{c}\text { LATER_DI } \\
(\mathbf{N}=56) \\
\text { Media } \\
(\mathbf{D T})\end{array}$ & $\begin{array}{c}\text { LATER_ZUR } \\
\mathbf{( N = 4 )} \\
\text { Media } \\
\text { (DT) }\end{array}$ & $\begin{array}{c}\text { Diferencia } \\
\text { de medias }\end{array}$ & $\begin{array}{c}\text { Test } \\
\text { univariante }\end{array}$ \\
\hline CREATIVIDAD & 11.45 & 10.25 & 1.2 & $\mathrm{t}=0.482$ \\
& $(4.678)$ & $(6.652)$ & & $\mathrm{p}=0.632$ \\
\hline INT_MUSI & 4 & 4.5 & -0.5 & $\mathrm{t}=-0.913$ \\
& $(1.062)$ & $(1.000)$ & & $\mathrm{p}=0.365$ \\
\hline
\end{tabular}

LATER_DI es la lateralidad diestra.

LATER_ZUR es la lateralidad zurda.

CREATIVIDAD es la variable que muestra la creatividad, medida mediante el test de inteligencia creativa (CREA). El valor de esta variable resulta de la suma de un total de 20 preguntas que podían plantear los/as alumnos/as en función de la visualización de una imagen.

INT_MUSI es la variable que refleja la inteligencia musical, medida mediante el test de inteligencia musical de las inteligencias múltiples de Gardner (1983). Concretamente, se plantearon un total de 5 preguntas de índole musical y el total de síes acumulados daba lugar al resultado final de esta variable.

$\mathbf{N}$ nos indica el número de alumnos que forman la muestra.

$(p)=p$-value.

De los resultados de la tabla 3 se desprende que la diferencia de medias para la variable creatividad es positiva (1.2), pero no es estadísticamente significativa, ya que el $p$-value es 0.632 , valor que está muy por encima de .05. Así pues, estos resultados sugieren que los alumnos con lateralidad diestra, en media, son más creativos que los alumnos con lateralidad zurda. Con respecto a la variable inteligencia musical (INT_MUSI), también se puede observar que la diferencia de medias entre alumnos con lateralidad diestra (4) y alumnos con lateralidad zurda (4.5) es negativa (-0.5), aunque tampoco es estadísticamente significativa, al arrojar el p-value un valor de 0.365 , superior a .05. Por tanto, en este caso los resultados sugieren que los alumnos con lateralidad diestra, en media, tienen una inteligencia musical ligeramente inferior que los alumnos con lateralidad zurda. 
En la tabla 4 presentamos las diferencias de medias que se ponen de manifiesto para la variable creatividad en base al rendimiento académico musical. Para ello se han creado dos grupos: uno formado por aquellos/as alumnas/os que presentan un rendimiento académico alto (REND_ACD_ALTO) y un rendimiento académico bajo (REND_ACD_BAJO), con el propósito de conocer si los alumnos con una mayor puntuación en creatividad obtienen mejores resultados académicos.

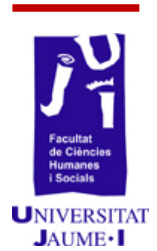

Tabla 4. Diferencia de medias para la variable creatividad en base al rendimiento académico

\begin{tabular}{|c|c|c|c|c|}
\hline Variable & REND_ACD_ALTO & REND_ACD_BAJO & Diferencia \\
de & $\begin{array}{c}\text { Media } \\
\text { (DT) }\end{array}$ & $\begin{array}{c}\text { Media } \\
\text { Media } \\
\text { (DT) }\end{array}$ & $\begin{array}{c}\text { Test } \\
\text { medias }\end{array}$ & \\
\hline CREATIVIDAD & 12.38 & 10.69 & 1.69 & $\mathrm{t}=-1.346$ \\
& $(4.771)$ & $(4.714)$ & & $\mathrm{p}=0.183$ \\
\hline
\end{tabular}

CREATIVIDAD es la variable que muestra la creatividad, medida mediante el test de inteligencia creativa (CREA). El valor de esta variable resulta de la suma de un total de 20 preguntas que podían plantear los/as alumnos/as en función de la visualización de una imagen.

REND_ACD es el rendimiento académico en música que han obtenido los alumnos en el tercer trimestre de este curso lectivo, medido mediante la nota obtenida en el tercer trimestre que puede oscilar entre 0 y 10 . Esta variable se ha diferenciado entre rendimiento académico alto y bajo, y se entiende que obtienen un rendimiento académico bajo si oscila entre $0 \mathrm{y}$ 6.99 y un rendimiento académico alto entre 7 y 10.

$\mathbf{N}$ nos indica el número de alumnos que forman la muestra.

$(p)=p$-value.

De los resultados de la tabla 4 se aprecia que la diferencia de medias para la variable creatividad es positiva (1.69), pero no es estadísticamente significativa, ya que el $p$-value es 0.183 , valor que está por encima de .05 . Por tanto, tenemos que rechazar la primera parte de la segunda hipótesis. Así pues, los resultados sugieren que los alumnos con mayor rendimiento académico, obtienen una mayor puntuación en creatividad, ya que la creatividad media es 12.38 en comparación con la media que obtienen los alumnos con rendimiento académico bajo que asciende a 10.69 .

En la tabla 5 presentamos las diferencias de medias que se pone de manifiesto para la variable rendimiento académico musical (REND_ACD) en base a la lateralidad manual izquierda. Para ello se han creado dos grupos: uno formado por aquellos/as alumnas/os que presentan una lateralidad diestra y otro por aquellos que presentan una lateralidad zurda. Con ello se pretende valorar si los alumnos con una lateralidad manual izquierda obtienen mejores resultados académicos. 

manual izquierda

\begin{tabular}{|c|c|c|c|c}
\hline Variable & $\begin{array}{c}\text { LATER_DI } \\
(\mathbf{N}=56) \\
\text { Media } \\
(\mathbf{D T})\end{array}$ & $\begin{array}{c}\text { LATER_ZUR } \\
(\mathbf{N}=\mathbf{4}) \\
\text { Media } \\
(\mathbf{D T})\end{array}$ & $\begin{array}{c}\text { Diferencia } \\
\text { de medias }\end{array}$ & $\begin{array}{c}\text { Test } \\
\text { univariante }\end{array}$ \\
\hline REND_ACD & 6.48 & 7.50 & -1.02 & $\mathrm{t}=-0.700$ \\
& $(1.307)$ & $(2.887)$ & & $\mathrm{p}=0.533$ \\
\hline
\end{tabular}

LATER_DI es la lateralidad diestra.

LATER_ZUR es la lateralidad zurda.

REND_ACD es el rendimiento académico en música que han obtenido los alumnos en el tercer trimestre de este curso lectivo, medido mediante la nota obtenida en el tercer trimestre que puede oscilar entre 0 y 10.

$\mathbf{N}$ nos indica el número de alumnos que forman la muestra.

$(p)=p$-value.

Del análisis de la tabla 5, se puede observar que la diferencia de medias para la variable rendimiento académico musical en función de si el alumno es diestro o zurdo es negativa (-1.02), pero no es estadísticamente significativa, dado que el $p$-value es 0.533 , valor que se sitúa por encima de .05. La segunda parte de la segunda hipótesis también se tiene que rechazar y a tenor de los resultados, se puede sugerir que los alumnos con lateralidad diestra obtienen un resultado académico musical medio (6.48) inferior que el que obtienen los alumnos con lateralidad zurda(7.50).

La tabla 6, muestra los resultados obtenidos para la regresión lineal, que nos permitirá contrastar empíricamente la tercera hipótesis, en la que se plantea que el tipo de lateralidad y creatividad conjuntamente predicen los resultados académicos musicales. 
Tabla 6. Resultado de la regresión lineal

\begin{tabular}{|c|c|c|}
\hline \multirow{2}{*}{ Variable } & \multicolumn{2}{|c|}{$\begin{array}{l}\text { Modelo } \\
\text { REND_ACAD }\end{array}$} \\
\hline & $\beta$ & $\begin{array}{l}\mathrm{t} \\
(p)\end{array}$ \\
\hline Constante & 6.906 & $\begin{array}{c}8.500 * * * \\
(0.000)\end{array}$ \\
\hline LATER & -1.087 & $\begin{array}{l}-1.479 \\
(0.145)\end{array}$ \\
\hline CREATIVIDAD & .058 & $\begin{array}{c}1.495 \\
(0.141) \\
\end{array}$ \\
\hline $\begin{array}{l}R^{2} \\
F(p)\end{array}$ & \multicolumn{2}{|c|}{$\begin{array}{c}6.8 \% \\
2.080(0.000)^{* * *}\end{array}$} \\
\hline
\end{tabular}

REND_ACD es el rendimiento académico en música que han obtenido los alumnos en el tercer trimestre de este curso lectivo, medido mediante la nota obtenida en el tercer trimestre que puede oscilar entre 0 y 10.

LATER es la variable que expresa la lateralidad, medida mediante el test de dominancia lateral de Harris (1993). Concretamente, si la dominancia lateral es diestra la variable toma el valor 1 y si es zurda toma el valor 0 .

CREATIVIDAD es la variable que muestra la creatividad, medida mediante el test de inteligencia creativa (CREA). El valor de esta variable resulta de la suma de un total de 20 preguntas que podían plantear los/as alumnos/as en función de la visualización de una imagen.

$(p)=p$ value.

El modelo analiza la capacidad predictiva de la lateralidad y la creatividad sobre el rendimiento académico. Así pues, vamos a estudiar si la lateralidad y creatividad conjuntamente influyen en el rendimiento académico musical del alumno. Si observamos los resultados del modelo que arroja la tabla 6 , se puede apreciar que el $R^{2}$ es un $6.8 \%$, indicando que un $6.8 \%$ de la varianza en rendimiento académico musical es explicada por la lateralidad y creatividad, mientras que el $93.2 \%$ restante es explicado por otros factores diferentes a la lateralidad y creatividad. Por otra parte, se observa que el modelo es estadísticamente significativo al 1\% ( $F=2.080$; $\mathrm{p}<0.000$ ). Por otra parte, se observa que la variable lateralidad (LATER) arroja un signo negativo $(\beta=-1.087)$, pero no es estadísticamente significativa $(p>0.05)$. Así pues, a tenor de este resultado podemos concluir que la lateralidad no parece predecir el rendimiento académico musical. Ahora bien, aunque este resultado no es estadísticamente significativo, sugiere que la lateralidad diestra repercute negativamente en el rendimiento académico musical, en línea con lo revelado previamente para la segunda parte de la segunda hipótesis, donde concluíamos que los alumnos con lateralidad diestra obtenían, en media, un resultado 
académico musical inferior que los alumnos con lateralidad zurda. Por otra parte, la variable creatividad presenta un signo positivo $(\beta=.058)$, pero tampoco es estadísticamente significativa $(p>0.05)$. Por lo tanto, tenemos que llegar a la conclusión que la creatividad tampoco influye en el rendimiento académico musical, tal y como habíamos predicho. Este resultado, aunque no significativo, también está en línea con lo revelado en la primera parte de la segunda hipótesis, donde se sugería que los alumnos con mayor creatividad obtienen un mayor rendimiento académico musical. Así pues, en base a estos resultados tampoco podemos aceptar la tercera hipótesis, y por consiguiente, tenemos que concluir que la lateralidad y la creatividad no influyen en el rendimiento académico musical.

\section{Discusión y Conclusiones}

La motivación intrínseca que se propone en esta investigación, principalmente por los profesionales e investigadores de la comunidad educativa, es conocer qué aspectos pueden intervenir en la mejora del rendimiento académico en música. Por ello, el objetivo principal de este trabajo es estudiar la relación entre la creatividad y el rendimiento académico musical. De este objetivo principal, se derivan dos objetivos específicos: estudiar la relación existente entre la creatividad con la lateralidad cerebral por un lado, y con la inteligencia musical por el otro, y examinar la relación entre la creatividad, la lateralidad y los resultados académicos en música. Para alcanzar estos objetivos concretos, se han planteado tres hipótesis. En la primera se predice que hay una relación directa y positiva entre la creatividad, la lateralidad manual izquierda, y la inteligencia musical, en la segunda hipótesis se plantea que los alumnos con mejores resultados académicos, obtendrán una mayor puntuación en creatividad y una lateralidad manual izquierda y en la tercera y última hipótesis, proponemos que el tipo de lateralidad y la creatividad conjuntamente van a predecir el nivel de resultados académicos. Este contribuye a proporcionar información sobre qué características neuropsicológicas, personales y formativas de los/as alumnos/as pueden influir en el rendimiento académico musical.

Los resultados de este trabajo nos llevan a rechazar las tres hipótesis planteadas, ya que ninguno de ellos han resultado ser estadísticamente significativos. Así pues, podemos concluir que la creatividad no explica ni la lateralidad cerebral ni la inteligencia musical así como tampoco el rendimiento académico musical. Igualmente, también se puede confirmar que los alumnos con mejores resultados académicos en música no obtienen ni mayor puntuación en creatividad ni en lateralidad manual izquierda. Finalmente, se concluye la escasa, por no decir casi nula, capacidad predictiva que tiene la lateralidad sobre el grado de creatividad y sobre el nivel de rendimiento académico musical.

Los resultados de este estudio tienen implicaciones académicas y educativas. Centrándonos en las implicaciones académicas, los resultados 
de este trabajo revelan que es necesario seguir investigando qué otros factores, diferentes de la creatividad y la lateralidad e inteligencia musical, influyen en el rendimiento académico musical. Algunos de estos factores podrían ser la psicomotricidad y la inteligencia, entre otros. Asimismo, y a tenor de los resultados obtenidos, también es necesario seguir estudiando otros factores diferentes a la creatividad, que podrían repercutir en la lateralidad cerebral y en la inteligencia musical. Además, se deberían estudiar la creatividad, la lateralidad y la inteligencia musical en alumnos de diferentes niveles educativos, desde la educación infantil hasta la educación secundaria, con el propósito de poner de manifiesto que resultados arroja. Finalmente, se debería considerar también la utilización de diferentes test para la medición de las variables así como el tipo de agrupamiento que realizamos, es decir, utilizar metodologías diferentes a las empleadas en este trabajo.

Las implicaciones a nivel educativo serían realizar una evaluación, después de la aplicación del programa de intervención, con la intención de conocer la funcionalidad y fiabilidad de dicho programa, así como para mejorar su contenido y desarrollo de dicho programa de intervención.

Este trabajo presenta algunas limitaciones. En primer lugar, nos gustaría destacar que la muestra utilizada en este estudio ha sido de un total de 60 alumnos/as de un colegio de educación primaria. Probablemente, con una muestra más grande de alumnos, los resultados revelados podrían haber sido diferentes. En segundo lugar, hay que indicar que el tiempo facilitado por el colegio donde se llevó a cabo el estudio fue limitado, y por tanto, los resultados se han visto condicionado por ello. Finalmente, señalar que no hemos estudiado todos los factores que podrían explicar el rendimiento académico musical.

\section{Bibliografía}

Alberdi, A., Alsina, M., Alsina, P., Arriaga, C., Flores, S., Giráldez, A., Ibarretxe, G., Loizaga, M., Ramírez de loaysa, A. (2010). Música: Complementos de formación disciplinar. Formación del profesorado. Barcelona: Grao.

BANICH, M. (1993). The neural bases of mental function. New York. Alexandria.

CostA, A. L. (Ed.). (1991). Developing minds. Vol.1. Washington: ASCD.

DAVIDOV, V.V. (1988). La enseñanza escolar y el desarrollo psíquico. Moscú: Editorial Progreso.

Ferranti, D., Perry, G,E., Gill, I., Guasch, J.l., Manoley, W.F., SanchezPARAMO, C., SCHADY, N. (2003). Closing the gap in Education and technology. Whashington: World Bank Latin American and Caribean Studies

Ferrándiz, G., Prieto, Mạ. D., Ballester, P. y Bermejo, Mạ. R. (2004). Validez y fiabilidad de los instrumentos de evaluación de las inteligencias múltiples en los primeros niveles instruccionales. Psicotema, 16 (1). 
FerRe, J., y IRABAU, E. (2002). El desarrollo neurofuncional del niño y sus trastornos. Madrid: Lebón.

Ferré, J. Casaprima, V. Catalán, J. y Mombiela, J. V. (2000). El desarrollo de la lateralidad infantil. Niño diestro- niño zurdo. Barcelona: Lebón.

FERREIRO, R. (1995). Educación para el talento. Guadalajara: SEP Jalisco.

FERreiro, R. (1999). A successful program in bilingual Education: ELI method. Executive report.

FerReIRo, R. (2003). Estrategias didácticas del aprendizaje cooperativo. Una nueva forma de enseñar y aprender: el constructivismo social. México: Trillas.

Ferreiro, R. (2012). La pieza clave del rompecabezas del desarrollo del la creatividad: la escuela. Revista Iberoamericana sobre la calidad, eficacia y cambio en Educación, 10 (2).

Feldhusen, J.F., y TRefFingeR, D.J. (1976). Design and evaluation of a workshop on creativity and problem solving for teachers. Journal of Creative Behavior, 10, 12-14.

Francisco, J., y RodríGUez, M. (2011). Contributions of neuroscience to the Understanding of Human creativity. Arte, Individuo y Sociedad, 23 (2), 4554.

Gaser, Ch., y Schlaug, G. (2003). Brain Structures differ Between Musicians And Non-Musicians. The Journal of Neuroscience, 23 (27): 9240-9245.

López, O., y Navarro, J. (2010). Creatividad e inteligencia: Un estudio en Educación Primaria. Revista de Investigación Educativa, 28 (2), 283-296

MARín, R. (1998). Creatividad y reforma educativa. Universidad de Santiago de Compostela.

MARTín, M.P. (2012). Apuntes de la asignatura de lateralidad. Rioja: UNIR.

NAVARRO, R. (2003). El rendimiento académico: concepto, investigación y desarrollo. Revista Iberoamericana sobre la calidad, eficacia y cambio en Educación, 1 (2).

OECD. (1998). Education at a glance. OECD Indicators. Paris: Organization for Economic Cooperation and Development.

Prensky, M. (2010). Teaching digital natives. Partnering for real learning. Thousand Oaks, CA: Corwin.

Portellano, J.A. (1992). Introducción al estudio de las asimetrias cerebrales.Coleccion Neurociencia. Madrid: Editorial CEPE.

PEÑA, J. (2007). Neuropsicologia de la conducta y Neuropsicologia. Madrid: Editorial Medica Panamericana.

RuIz, J. I. (2012). Metodología de la investigación cualitativa. Universidad de Deusto. Serie de Ciencias Sociales, Vol.15. 
SERRA, J. (2012). Introducción a la creatividad. Dialnet OAl Boletín de Información. №. 325, (87-104)

SÁNCHEZ, S., y EPELDE A. (2013). Análisis del tratamiento educativo de la creatividad por medio de la música en aulas de educación primaria con amplia diversidad étnica y cultural. European Scientific Journal, 34 (9).

Schellenberg, E.G. ( 2004). Music and cognitive Abilities. American Psicological Society, 14, (2).

TAPSCOTT, D., y WILLIAMS, A, D. (2006). Wikinomics. La nueva economía de las multitudes inteligentes. Barcelona: Paidós.

ToRRANCE, E. P. (1960). Explorations in creative talent. Education Leadership, 20, 7-10. 